NOTA CIENTÍFICA

\title{
APROPRIAÇÃO E ANÁLISE DE CUSTO DE IMPLANTAÇÃO DE POMAR DE PESSEGUEIRO
}

\section{APPROPRIATION AND ANALYSIS OF COST OF IMPLANTATION OF PEACH ORCHARD}

\author{
Joel Ferreira PENTEADO JUNIOR ${ }^{1}$ \\ Louise Larissa MAY-DE-MIO² \\ Lino Bittencourt MONTEIRO ${ }^{3}$ \\ Waldomiro GAYER NETO 4
}

\begin{abstract}
RESUMO
Este trabalho foi realizado no município de Araucária, localizado na região metropolitana de Curitiba, PR. Os objetivos foram apresentar cálculos e análise dos custos de implantação em pomares de pessegueiro, e também, construir e disponibilizar uma planilha eletrônica contendo coeficientes técnicos e valores, que permita as mais diferentes substituições dos valores e indicadores, para ser utilizada como uma ferramenta para a gestão de estabelecimentos agrícolas. Os custos de implantação e formação de 1,0 ha da cultura, contemplando até o primeiro ano de plantio, atingiram $R \$ 8849,24$ ha, onde os componentes mais significativos na composição dos custos foram, os gastos com mudas e serviços, representando $87,67 \%$ do total. Individualmente, o número de plantas por hectare tem grande importância na composição dos custos. Neste caso representou, $56,50 \%$ do total.
\end{abstract}

Palavras-chave: custos; planilha eletrônica; fruticultura; tecnologia.

\begin{abstract}
This work was carried through in the city of Araucária, located in the region metropolitan of Curitiba, PR. The objectives were present calculations and analysis of the costs of implantation in peach orchards, and also, to construct and to offer an spreadsheet detailed with coefficients technician and values, that allow to the most different substitutions of the values and pointers, thus making possible to personalize each in case that, to be used as a tool for the management of agricultural establishments. The implantation and formation costs of 1,0 ha of the culture, contemplating until the first year of plantation, had reached $\mathrm{R} \$ 8849,00$ where the most significant components in the composition of the costs were, the cost of seedlings and services, representing $87.67 \%$ of the total. Individually, the number of plants for hectare has great importance in the composition of the costs for hectare and in this in case that it represented, $56.50 \%$ of the total.
\end{abstract}

Key-words: costs; technology appraisal; fruit production; technology. 


\section{INTRODUÇÃO}

No Brasil cerca de 30 mil produtores de frutas exploram uma área de 58,8 mil ha, o que representam $2,9 \%$ do valor bruto da produção (CNA, 2006). Para a EMBRAPA (2006), a fruticultura, no Brasil, gera um PIB de US $\$ 1,5$ bilhão. Segundo a SEAB/DERAL (2006), a produção paranaense de prunáceas, foi de 32,6 mil t, em 2004. O maior produtor de pêssego do Paraná é o município de Lapa, com produção anual de 5040 t (IPARDES, 2006). Araucária é o segundo, com uma produção anual de $940 \mathrm{mil}$ t, em uma área de 360 ha (IPARDES, 2006).

A exploração racional dos pomares de pêssegos depende de um conjunto de fatores que podem afetar o desempenho produtivo e a rentabilidade, tais como: condução do pomar e relação de preços entre insumos e produto (MEDEIROS, 1998) e necessitam de altos investimentos para implantação e, em cultivos não adensados, apresentam, normalmente, retornos financeiros significativos somente a partir do quinto ano (EMBRAPA, 2006).

Conforme FETT (2000), a manutenção anual do pomar, possui um custo considerável, que pode inviabilizar a cultura se não for controlado. Devido a essa característica, o conhecimento dos custos e dos sistemas de produção de pêssegos passam a ser importantes na tomada de decisão para a otimização fatores de produção (MADAIL, 2002).

O presente trabalho objetivou apresentar cálculos e análise dos custos de implantação em pomares de pessegueiro, e também, construir e disponibilizar uma planilha eletrônica detalhada, que permita as mais diferentes substituições dos valores e indicadores, possibilitando assim, personalizar cada caso.

\section{METODOLOGIA}

O estudo foi conduzido em um estabelecimento agrícola, no município de Araucária, PR, localizado a 492 25' $24^{\prime \prime}$ de longitude oeste e 25응 37 ' 03 " de latitude sul, tendo uma altitude média de $975 \mathrm{~m}$ (SEAB, 2007).

A determinação dos custos, no presente estudo, foi apropriada individualmente, baseada nas atividades agronômicas, conforme demonstrado por DI DOMENICO (1994) e POMPERMEYER (1999). Este método foi escolhido por avaliar com precisão as atividades desenvolvidas e identifica as despesas necessárias para a sua realização.

Para registro e determinação dos custos, foram elaboradas planilhas em plataforma Microsoft

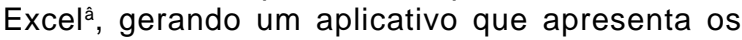
custos de implantação de 1,0 ha de pomar de pessegueiro, contemplando parâmetros de gerenciamento dos custos, tais como: quantidades dos direcionadores de custo, custo unitário, porcentual de participação dos coeficientes na atividade e os gastos envolvidos em cada componente da estrutura de custos divididos em alguns grandes grupos, assim distribuídos:

a) Serviços: são discriminados os custos com mão-de-obra e mecanização. A unidade utilizada para mensurar a quantidade de mão-de-obra empregada em determinada atividade é dia-homem (dh). A utilização de máquinas é medida por horasmáquina $(\mathrm{hm})$, correspondendo ao número de horas necessárias para uma máquina realizar uma operação. Para a estimativa das horas/máquina, é utilizada a classificação tradicional onde são considerados os valores médios de salário do tratorista, consumo e preços médios de combustíveis e lubrificantes, e a depreciação média. Todos os valores são referentes a um trator de $75 \mathrm{cv}$ com rodas de pneus:

b) Insumos: são contemplados os custos com fungicidas, fertilizantes e inseticidas necessários para o primeiro ano de vida do pomar;

c) Outros insumos: envolve gastos com administração, mudas, caixas e sacolas de colheita, análises foliar e de solos, armadilhas para monitoramento de insetos;

d) Administrativos: incluem as despesas administrativas e as realizadas com manutenção e depreciação de implementos e equipamentos. Os dispêndios com manutenção, são os valores necessários para manter os bens, como máquinas e implementos, em plena condição de uso. Os cálculos destes valores são feitos por meio de uma estimativa baseada na média histórica da intensidade de uso dos respectivos bens na propriedade estudada e transformando-a em valor percentual que incide sobre o custo total.

Foram consideradas apenas as despesas diretas, isto é, aquelas diretamente relacionadas com a produção, não sendo incluídos, o valor de remuneração da terra, os juros sobre o capital empregado e os aportes financeiros para custeio ou investimentos.

Os coeficientes técnicos e os indicadores foram obtidos por meio de entrevista direta com o proprietário responsável pela administração do estabelecimento, e ainda junto a empresas comerciais ligadas a insumos agrícolas na região.

\section{RESULTADOS E DISCUSSÃO}

Analisando os dados referentes ao item "serviços" (Tabela 1), verifica-se que as atividades de capina manual e poda de formação apresentam maior percentagem de gastos em relação ao indicador "mão-de-obra", sendo respectivamente de 6,78 e $3,84 \%$. Enquanto que a atividade com enxada rotativa é a que tem maior peso no indicador "mecanização", com 4,07\% em relação à totalidade dos custos. Esta situação é decorrente do manejo de ervas invasoras, onde foi racionalizado o uso de herbicidas, optando-se pela a capina na linha e entre plantas, valorizando as práticas de cultivo recomendadas nas normas de produção integrada de frutas (FACHINELLO et al., 2003). 
PENTEADO JUNIOR, J.F. et al. Apropriação e análise de custo de...

TABELA 1 - Custos de serviços com mão-de-obra e mecanização para de implantação de 1,0 ha de pomar de pessegueiro em Araucária, PR. 2006.

\begin{tabular}{|c|c|c|c|c|c|}
\hline \multirow{2}{*}{ Discriminação } & \multirow[t]{2}{*}{ Unidade $^{1}$} & \multirow{2}{*}{$\begin{array}{c}\text { Preço Unidade } \\
\mathrm{R} \$\end{array}$} & \multicolumn{2}{|c|}{ Custos no $1^{\circ}$ ano } & \multirow{2}{*}{ Participação } \\
\hline & & & Quantidade & Valor $\mathrm{R} \$$ & \\
\hline \multicolumn{6}{|l|}{ Mão-de-obra (1) } \\
\hline Abertura de covas e plantio & $d / h$ & 20,00 & 10,0 & 200,00 & $2,26 \%$ \\
\hline Aplicação insetic.e fungicidas & $d / h$ & 20,00 & 3,0 & 60,00 & $0,68 \%$ \\
\hline Aplicação de fertilizantes & $d / h$ & 20,00 & 3,0 & 60,00 & $0,68 \%$ \\
\hline Aplicação de calcário & $d / h$ & 20,00 & 6,0 & 120,00 & $1,36 \%$ \\
\hline Capina Manual & $d / h$ & 20,00 & 30,0 & 600,00 & $6,78 \%$ \\
\hline Coleta de solo $\mathrm{p} /$ análise & un & 20,00 & 1,0 & 20,00 & $0,23 \%$ \\
\hline Combate a Formiga & $d / h$ & 20,00 & 5,0 & 100,00 & $1,13 \%$ \\
\hline Limpeza e roçada & $d / h$ & 20,00 & 2,0 & 40,00 & $0,45 \%$ \\
\hline Marcação do terreno & $d / h$ & 20,00 & 0,5 & 10,00 & $0,11 \%$ \\
\hline Poda de formação & $d / h$ & 20,00 & 17,0 & 340,00 & $3,84 \%$ \\
\hline Roçada entre-linhas & $d / h$ & 20,00 & 4,0 & 80,00 & $0,90 \%$ \\
\hline Sub-total mão-de-obra & & & & 1630,00 & $18,42 \%$ \\
\hline \multicolumn{6}{|l|}{ Mecanização (2) } \\
\hline Aplicação insetic e fungicidas & $\mathrm{h} / \mathrm{m}$ & 30,00 & 9,0 & 270,00 & $3,05 \%$ \\
\hline Aplicação de fetilizantes & $\mathrm{h} / \mathrm{m}$ & 30,00 & 2,0 & 60,00 & $0,68 \%$ \\
\hline Aração & $\mathrm{h} / \mathrm{m}$ & 30,00 & 0,0 & - & $0,00 \%$ \\
\hline Distribuição de mudas & $\mathrm{h} / \mathrm{m}$ & 30,00 & 1,5 & 45,00 & $0,51 \%$ \\
\hline Enxada Rotativa & $\mathrm{h} / \mathrm{m}$ & 30,00 & 12,0 & 360,00 & $4,07 \%$ \\
\hline Gradagem & $\mathrm{h} / \mathrm{m}$ & 30,00 & 0,0 & - & $0,00 \%$ \\
\hline Roçada entre-linhas & $\mathrm{h} / \mathrm{m}$ & 45,00 & 6,0 & 270,00 & $3,05 \%$ \\
\hline Roçada na linha & $\mathrm{h} / \mathrm{m}$ & 30,00 & 0,0 & - & $0,00 \%$ \\
\hline Subsolagem (bico-de-pato) & $\mathrm{h} / \mathrm{m}$ & 41,00 & 3,0 & 123,00 & $1,39 \%$ \\
\hline Sub-total Mecanização & & & & 1128,00 & $12,75 \%$ \\
\hline Sub-total Serviços $(1+2)$ & & & & 2758,00 & $31,17 \%$ \\
\hline
\end{tabular}

${ }^{1} \mathrm{~d} / \mathrm{h}$ : dias/homem e h/m: horas/máquina

Os custos dos insumos (Tabela 2), o maior dispêndio de gastos é com fertilizantes $(8,70 \%)$. Nesta fase, os custos com controle de pragas e doenças não são muito significativos, correspondendo a $1,29 \%$ do custo total, situação que seria interessante para toda a vida do pomar, pois conforme MOLINARI (2001) é recomendável que a aplicação de agrotóxicos seja feita somente nos períodos recomendados ou quando as pragas atingirem o nível de controle. Além de promover a redução nos custos com agroquímicos, observamse benefícios ambientais, preservando a biodiversidade e a saúde do produtor e do consumidor (BOTTON et al., 2001; FARIAS et al., 2004). Além disso, o uso abusivo de pulverizações pode aumentar o risco de resistências às principais pragas.
Quanto aos dispêndios com outros insumos (Tabela 3), destaca-se a despesa com mudas, representando $56,50 \%$ em relação ao custo total. Este valor é determinado pelo espaçamento adotado no pomar estudado, que é de 5,0 × 1,8 m, totalizando 1111 plantas ha ${ }^{-1}$.

Os custos administrativos (Tabela 4) foram apropriados pelo sistema de rateio e apresentaram $1,96 \%$ do custo total de implantação.

Uma síntese dos resultados encontrados (Tabela 5) nos mostra que o custo total de implantação de 1,0 ha de pomar de pessegueiro foi de $R \$ 8849,24$. Os dados obtidos nos mostram que o percentual de gastos com "outros custos", mãode-obra e mecanização, são os mais significativos. Nos demais itens, as percentagens são relativamente bem distribuídas. 
PENTEADO JUNIOR, J.F. et al. Apropriação e análise de custo de...

TABELA 2 - Custos com insumos para implantação de 1,0 ha de pomar de pessegueiro em Araucária, PR.

\begin{tabular}{|c|c|c|c|c|c|}
\hline \multirow[t]{2}{*}{ Discriminação } & \multirow[t]{2}{*}{ Unidade } & \multirow{2}{*}{$\begin{array}{l}\text { Preço Unidade } \\
\mathrm{R} \$\end{array}$} & \multicolumn{2}{|c|}{ Custos no $1^{\circ}$ ano } & \multirow[t]{2}{*}{ Participaçãc } \\
\hline & & & Quantidade & Valor $\mathrm{R} \$$ & \\
\hline Fungicidas (1) & & & & & $0,00 \%$ \\
\hline Calda bordalesa & 1 & 12,50 & 0,0 & - & $0,00 \%$ \\
\hline Calda sulfocálcia & 1 & 1,49 & 0,0 & - & $0,00 \%$ \\
\hline Captana (Orthocide) & $\mathrm{kg}$ & 20,00 & 0,0 & - & $0,00 \%$ \\
\hline Cercobin & 1 & 48,00 & 0,0 & - & $0,00 \%$ \\
\hline Mancozebe (Dithane) & $\mathrm{kg}$ & 18,00 & 0,6 & 10,80 & $0,12 \%$ \\
\hline Iprodiona (Rovral) & 1 & 74,20 & 0,5 & 37,10 & $0,42 \%$ \\
\hline Tebuconazole & 1 & 63,56 & 0,0 & - & $0,00 \%$ \\
\hline Sub-total & & & & 47,90 & $0,54 \%$ \\
\hline \multicolumn{6}{|l|}{ Inseticidas (2) } \\
\hline Amitraz (Acaricida) & 1 & 110,00 & & - & $0,00 \%$ \\
\hline Dimetoato & 1 & 10,90 & & - & $0,00 \%$ \\
\hline Enxofre (Kumulus) & $\mathrm{kg}$ & 5,90 & & - & $0,00 \%$ \\
\hline Fipronil (Formicida) & $\mathrm{kg}$ & 5,90 & & - & $0,00 \%$ \\
\hline Fenitrotion (Sumithion) & $\mathrm{kg}$ & 10,00 & 5,0 & 50,00 & $0,57 \%$ \\
\hline Supracid & 1 & 56,00 & 0,3 & 16,80 & $0,19 \%$ \\
\hline Triona & 1 & 49,00 & & - & $0,00 \%$ \\
\hline$\underline{\text { Sub-total }}$ & & & & 66,80 & $0,75 \%$ \\
\hline \multicolumn{6}{|l|}{ Fertilizantes (3) } \\
\hline Calcário & $\mathrm{t}$ & 50,00 & 6,0 & 300,00 & $3,39 \%$ \\
\hline Cloreto de Potássio & $\mathrm{kg}$ & 0,50 & & - & $0,00 \%$ \\
\hline NPK 8-20-20 & $\mathrm{kg}$ & 6,92 & 55,5 & 384,06 & $4,34 \%$ \\
\hline Superfosfato triplo & $\mathrm{kg}$ & 0,55 & & - & $0,00 \%$ \\
\hline Uréia & $\mathrm{kg}$ & 0,77 & 111,0 & 85,47 & $0,97 \%$ \\
\hline Sub-total & & & & 769,53 & $8,70 \%$ \\
\hline Sub-total Insumos $(1+2+3)$ & & & & 884,23 & $9,99 \%$ \\
\hline
\end{tabular}

TABELA 3 - Custos de outros insumos para implantação de 1,0 ha de pomar de pessegueiro em Araucária, PR. 2006.

\begin{tabular}{|c|c|c|c|c|c|}
\hline \multirow[t]{2}{*}{ Discriminação } & \multirow[t]{2}{*}{ Unidade } & \multirow{2}{*}{$\begin{array}{c}\text { Preço Unidade } \\
\mathrm{R} \$\end{array}$} & \multicolumn{2}{|c|}{ Custos no $1^{\circ}$ ano } & \multirow[t]{2}{*}{ Participação } \\
\hline & & & Quantidade & Valor $\mathrm{R} \$$ & \\
\hline Análise de solo & un & 20,00 & 1,0 & 20,00 & $0,23 \%$ \\
\hline Análise foliar & un & 20,00 & & - & $0,00 \%$ \\
\hline Gliphosato & I & 14,00 & 1,0 & 14,00 & $0,16 \%$ \\
\hline Mudas & un & 4,50 & $1.111,0$ & $4.999,50$ & $56,50 \%$ \\
\hline Sub-total Outros Insumos & & & & $5.033,50$ & $56,88 \%$ \\
\hline
\end{tabular}


TABELA 4 - Custos administrativos para implantação de 1,0 ha de pomar de pessegueiro em Araucária, PR. 2006.

\begin{tabular}{lc}
\hline Discriminação & Custos no 1ªno \\
\cline { 2 - 2 } Administração/escritório & Valor R\$ \\
Manutenção.Depreciação. Máq. Equipamentos & 86,76 \\
\hline Sub-total Custos Administrativos & $8,98 \%$ \\
\hline
\end{tabular}

TABELA 5 - Custos totais para implantação de 1,0 ha de pomar de pessegueiro em Araucária, PR. 2006.

\begin{tabular}{lrr}
\hline Discriminação & Valor R\$ & Participação \\
\hline Custo serviços & 2758,00 & $31,17 \%$ \\
Custo insumos & 884,23 & $9,99 \%$ \\
Outros Insumos & 5033,50 & $56,88 \%$ \\
Custos fixos & 173,51 & $1,96 \%$ \\
\hline Total & 8849,24 &
\end{tabular}

Os custos de produção podem variar em função do nível de tecnologia adotado e da disponibilidade de recursos, nos diferentes estabelecimentos rurais. No Estado de São Paulo, o custo de produção de pêssego foi de $R \$ 9694,00$ ha $^{-1}$ no primeiro ano (AGRIANUAL, 2007) e no Rio Grande do Sul, os custos de implantação totalizaram $\mathrm{R} \$ 7.513,00 \mathrm{ha}^{-1}$ (EMBRAPA, 2006).

A planilha desenvolvida neste trabalho permite simulações e serve para construção de cada caso em particular. Algumas atividades relacionadas na planilha apresentam-se sem valor, porque não foram utilizadas no caso estudado, mas podem ser adotadas quando da implantação de pomares de pêssego em outra situação. Todas as células componentes do aplicativo, que contêm as atividades, os indicadores e as despesas, são abertas para inclusão de qualquer dado, enquanto que as colunas "E" (valor), "F" (participação) e as linhas de subtotal e total, automaticamente calculam os valores, permitindo a sua adequação a cada caso.

O aplicativo eletrônico, em forma de planilha, permite o cálculo, tanto para sistemas que adotam a produção integrada de frutas (PIF), quanto para a produção convencional, bastando que sejam omitidos ou incluídos indicadores que façam parte do sistema escolhido. Estará disponível para downloading no site do Grupo de Ensino, Extensão e Pesquisa em Produção Integrada de Frutas GEEPPIF no seguinte endereço eletrônico: http:// www.geeppif.ufpr.br.

\section{CONCLUSÕES}

1) O aplicativo eletrônico, produto deste estudo, é uma ferramenta de fácil operação e permite o cálculo dos dispêndios necessários para a implantação de pomares de frutas.

2) Os componentes mais significativos na composição dos custos foram os gastos com mudas e serviços (mão-de-obra e mecanização). O espaçamento escolhido influencia diretamente no montante dos custos, pois determina o número de plantas por hectare e conseqüentemente o volume de insumos utilizados.

3) O método de determinação de custos baseado em atividades, mostrou-se muito útil, para a apropriação dos custos e poderá ser utilizado para a tomada de decisões e gestão na propriedade agrícola, quando da implantação de pomares de pêssego.

\section{REFERÊNCIAS}

1. AGRIANUAL 2007. Anuário da agricultura brasileira. São Paulo: IFNP Instituto Consultoria \& Comércio, 2007. 516 p.

2. BOTTON, M.; ARIOLI, C.J.; SCOZ, P.L. Situação atual e perspectivas para o manejo de pragas do pessegueiro no Sistema de Produção Integrada. In: ENCONTRO NACIONAL SOBRE FRUTICULTURA DE CLIMA TEMPERADO, 4., 2001, Fraiburgo. Anais. Caçador: EPAGRI, 2001. p. 110-115.

3. CONFEDERAÇÃO NACIONAL DAAGRICULTURA E PECUÁRIA. Fruticultura. Disponível em: <www.cna.org.br>. Acesso em: 30 set. 2006.

4. DI DOMENICO, G.B., LIMA, P.C. Activity based costing (ABC): uma nova ferramenta para gestão total dos custos. Máquinas e metais. São Paulo: Aranda Editora Técnica, 1994. 
PENTEADO JUNIOR, J.F. et al. Apropriação e análise de custo de...

5. EMPRESA BRASILEIRA DE PESQUISA AGROPECUÁRIA. Sistema de produção de pêssego. Disponível em: <www.cnpuv.embrapa.br/publica/sprod/PessegodeMesaRegiaoSerraGaucha/custos.htm>. Acesso em 15 nov 2006.

6. FACHINELLO, J.C.; COUTINHO, E.F.; MARONDIN, G.A.B.; BOTTON, M.; MAY-DE-MIO, L.L. Normas técnicas e documentos de acompanhamento da produção integrada de pêssego. Pelotas: Universidade Federal de Pelotas, Faculdade de Agronomia Eliseu Maciel, 2003. 95 p.

7. FARIAS, R.M.; MARTINS, R.C.; NUNES, J.L.S.; GUERRA, D.S.; ZANINI, C.; MARODIN, G.A.B. Produção convencional $x$ integrada em pessegueiro na Depressão Central do Rio Grande do Sul. Revista da Faculdade de Zootecnia Veterinária e Agronomia de Uruguaiana, v.10, p. 87-100, 2004.

8. FETT, M.S. Análise econômica de sistemas de cultivo de macieiras no município de Vacaria RS. Porto Alegre, 2000. 145 f. Dissertação (Mestrado em Economia Rural) - Curso de Pós-Graduação em Agronomia, Universidade Rural do Rio Grande do Sul.

9. GRAÇA, L.R.; RODIGHERI, H.R.; CONTO, A.J. de. Custos florestais de produção: conceituação e aplicação. Colombo: Embrapa Florestas, 2000. 32 p. (Embrapa Florestas. Documentos, 50).

10. IPARDES. Instituto Paranaense de Desenvolvimento Econômico e Social. Caderno Estatístico: Município de Araucária. Disponível em www.ipardes.gov.br/cadernos/Montapdf.php?Municipio=83700. Acesso em 10/09/2006.

11. MADAIL, J.C.M.; REICHERT, L.J.; DOSSA, D. Análise da rentabilidade dos sistemas empresarial e familiar de produção de pêssego no sul do Rio Grande do Sul. Pelotas: Embrapa Clima Temperado, 2002. 43 p. (Embrapa Clima Temperado. Documentos, 86).

12. MEDEIROS, A.R.M. Leguminosas de inverno: uma opção no manejo do solo em pomar de fruteiras de clima temperado. Hortisul, v. 2, n. 1, p. 14-15, 1998.

13. MOLINARI, F. La difesa dal fitofagi nella produzione integrata del pesco in Itália. In: SEMINÁRIO SOBRE PRODUCÃO INTEGRADA DE FRUTAS, 3., 2001, Bento Gonçalves. Anais. Bento Gonçalves: Embrapa Uva e Vinho, 2001. p. 48-58.

14. SEAB. Secretaria de Estado da Agricultura e do Abastecimento do Estado Paraná. Evolução da produção de frutas no Paraná. Disponível em: www.seab.pr.gov.br/arquivos/File/deral/efpr.xls. Acesso em 30 set 2007.

15. POMPERMAYER, C.B. Sistemas de gestão de custos: dificuldades na implantação. Revista FAE, v. 2, n. 3, p. 21-28, 1999.

Recebido em 02/08/2007

Aceito em 10/12/2007 
